\title{
Designing the reference architecture of human ecology on the basis of the methodology of the function oriented modelling
}

\author{
Svetlana Mukhametdinova ${ }^{1,{ }^{*}, \text { Evgenia Tyumentseva, Oleg Patlasov }}{ }^{1}$, and Oleg Luchko ${ }^{1}$ \\ ${ }^{1}$ Omsk Humanitarian Academy, 4-ya Chelyuskincev Str., 2A, 644105 Omsk, Russia \\ ${ }^{2}$ Omsk State Technical University, Prospect Mira Str., 11, 644050 Omsk, Russia
}

\begin{abstract}
The dynamic nature of changes in the conditions of existence and functioning of modern communities requires comprehensive and integrated research in order to develop a reference architecture of human ecology, as a system of scientifically substantiated criteria for a qualitative and quantitative assessment of the impact of the external environment on various aspects of human life and the formation of a balanced strategy for managing social development. The paper presents one of the approaches to the design of the reference architecture of human ecology based on the methodology of the function oriented modelling and provides examples of models developed in accordance with the IDEF0 notation. The study identified the main stages in the design of the reference architecture of human ecology from collecting information to developing software that makes it possible to automate the processing of input information characterizing various factors affecting the quality of life of the population to developing the architecture of human ecology in a particular region or city and comparing it with the reference one.
\end{abstract}

\section{Introduction}

Various aspects of human ecology have a significant impact on all spheres of society, from demography to economics, and in this regard, comprehensive research in this area is relevant. Since human ecology studies the patterns of interaction of human communities with the natural, social, industrial, ecological and hygienic factors surrounding them, a wide range of methods and approaches are used in the analysis of the set of factors that characterize its state.

There are various approaches to defining the range of issues related to human ecology, in particular, the broadest of them - the holistic approach - involves considering the following issues:

- population dynamics of the region, city, etc.;

- age and gender structure of society;

- the state of health of the population, which is characterized by the average life expectancy, the most common types of diseases and causes of death;

\footnotetext{
${ }^{*}$ Corresponding author: muhamet-m@yandex.ru
} 
- nutritional characteristics (quality and quantity of food products, nutritional value and calorie content of food, as well as methods of its preparation);

- dominant types of labor activity, the degree of mechanization of the production sector of the economy;

- types of energy sources used in the economy and everyday life;

- features of the settlement system and cultural and hygienic skills of people;

- the level of social and economic well-being and spiritual and moral development of the population;

- ecology of home and clothing;

- the state of the environment;

- efficiency of service sector management and consumer problems;

- the specificity of social interaction of people, including family relations, etc.

A number of scientific publications by scientists from different countries of the world are devoted to the study of problems of human ecology.

In the work of He V.Kh., Tkachenko I.N., Skripnichenko D.E., Dubinin A.V. the factors influencing the stability of the socio-ecological-economic system are analyzed and the problems of interaction between human society and nature, economy and ecology are described [1].

Article [2] is devoted to the analysis of problems that in the long term can lead to the emergence of an environmental crisis and threaten the survival and preservation of society if humanity does not take decisive action to protect the environment from the harmful effects of negative factors.

Features of the use of cognitive methods and models in the analysis and forecasting of various aspects of human ecology are considered in works [3, 4].

In article [5], an attempt is made to form a holistic view of emotional culture as the basis of a person's holistic health.

Kurbatova S. M., Aisner L. Yu., Volkova A. G. [6] consider the dualism of modern cities, which, on the one hand, have a large and varied potential, and on the other hand, they must ensure that the population implement their multiple needs. Based on the data of urban ecology and the technosphere, they are developing ways of new environmentally friendly urban planning by studying the mechanisms of interaction between the urbanized and natural environment.

In [7], a spatial model of urban development is presented by assessing and comparing a variety of compromise scenarios between ecological processes and urban expansion. The authors developed a conceptual framework for regional urban environmental sustainability with six stages: pattern recognition, landscape process, problem diagnosis, integrated ecological network, urbanization modeling, and decision support. Comparing urban growth in three scenarios, A. Cai, J. Wang, I. MacLachlanand, L. Zhu found that a compromise scenario based on preserving and restoring the environment (medium safety level) resolved conflicts more effectively, which limited urban expansion in an area of high ecological importance creating regional ecological networks.

Study [8] examines the issues of models, tools and rankings in the subject area of smart cities, which served as the basis for the standardization of the Smart City. The authors compare the proposed international standards with the approaches prevailing at present in domestic practice.

Experts have developed an approach to the design of the Smart City system using the methods of system dynamics and agent-based modeling [9], as well as on the basis of a synergetic approach that allows balancing the problems of developing intelligent technogenic systems [10]. In [11], a method is proposed for determining the criteria and parameters of the comfort of living. 
Thus, human ecology as a science, the field of study of which includes the interaction of a person as a representative of nature and a representative of society with all components of the environment, has a complex structure, and its individual components are characterized by many different qualitative and quantitative parameters, but there is no methodology that integrates interdisciplinary approaches (economic and mathematical, expert, cognitive, reflexive, etc.) and makes it possible to assess and predict changes in the degree of impact and, as a consequence, transformation of the state of human ecology on human communities. In addition, there is no reference architecture of human ecology, which would not only reflect the structural features of the problem area, but also include a methodology for identifying the relationships and the degree of mutual influence of various factors on the level of human ecology as a target factor, as well as methods for determining, obtaining, processing and interpretation of their qualitative and quantitative characteristics.

The development of the reference architecture is aimed at creating a methodology and tools that will make it possible to scientifically determine the level of the state of human ecology in various cities and regions based on the analysis of large arrays of heterogeneous data using cross-modeling methods and the capabilities of modern information technologies.

\section{Methods and types of the Earth's remote sensing (Materials and Methods)}

Work on the creation of the reference architecture of human ecology is a complex process that includes the development of methodological approaches, the choice of methods and modeling tools, as well as software implementation, which, in the future, will automate the most time-consuming stages of processing large arrays of data to determine the level of the state of human ecology. in a particular region.

When creating the reference architecture, it is advisable to use the function oriented approach to modeling business processes, since it allows not only to represent the processes under study in the form of a sequence of works that transform incoming flows of various etymologies (informational, material, financial) into outgoing ones, but also to design appropriate software [12].

For the functional approach to modeling, the main structural element is function (work). In turn, business processes are represented as an interconnected sequence of functions that transform input flows (informational or material) of the process into outputs through the use of certain resources. A distinctive feature of the functional modeling methodology is the distinction between the flows of data, materials, components, etc., and the work that transforms them. A set of notations or graphic elements and regulations for their use is the basis of the function oriented modeling.

Thus, the purpose of this approach is to build functional models of the system under study to describe all the necessary processes at a level of the detailed elaboration sufficient for unambiguous modeling of the problem area. The main advantages of function oriented modeling include a structural approach to design, procedural severity of decomposition, as well as high visibility of information presentation.

Nowadays, the most common notations for the functionoriented modeling for business processes are the IDEF0, IDEF3 and DFD standards.

Initially, the methodology under consideration was used in the design of information systems, but now it has found wide application for the analysis of business processes, since it makes it possible to structure and visualize their components for a deeper understanding of the features of the functioning of the system for developing effective management decisions. 
At the first stage of the study, a context diagram "Designing the reference model of human ecology" was built in accordance with the IDEF0 notation (Fig. 1).

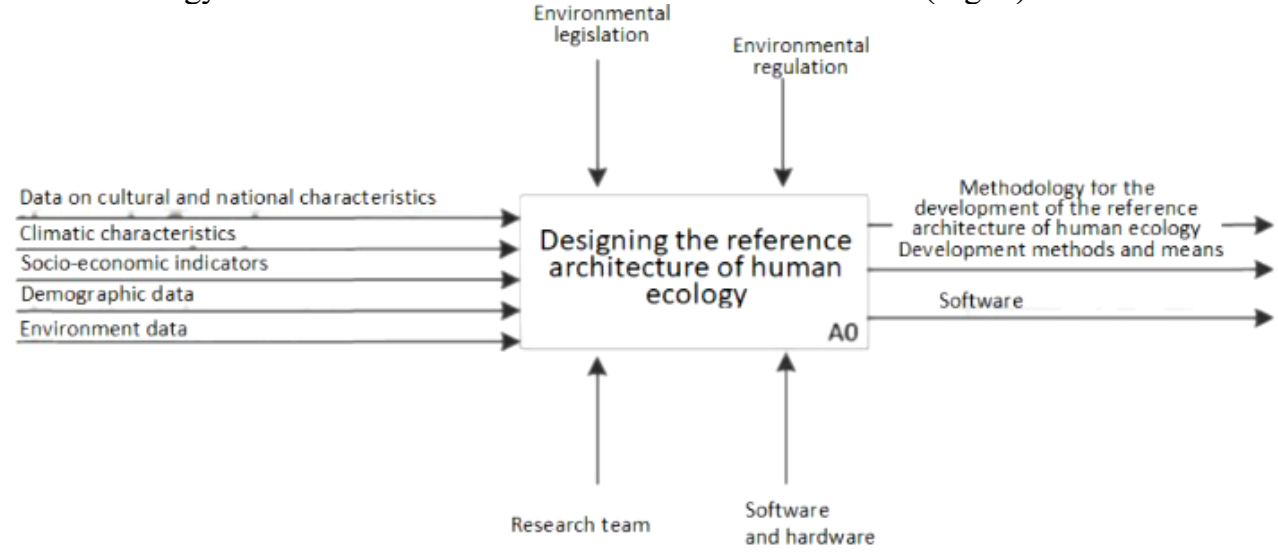

Fig. 1. Diagram "Designing the reference architecture of human ecology".

Further, a decomposition of the context diagram was developed, which presents the detailing of the design process of the reference architecture of human ecology from analysis and structuring of input information to computer implementation and constructed models (Fig. 2).

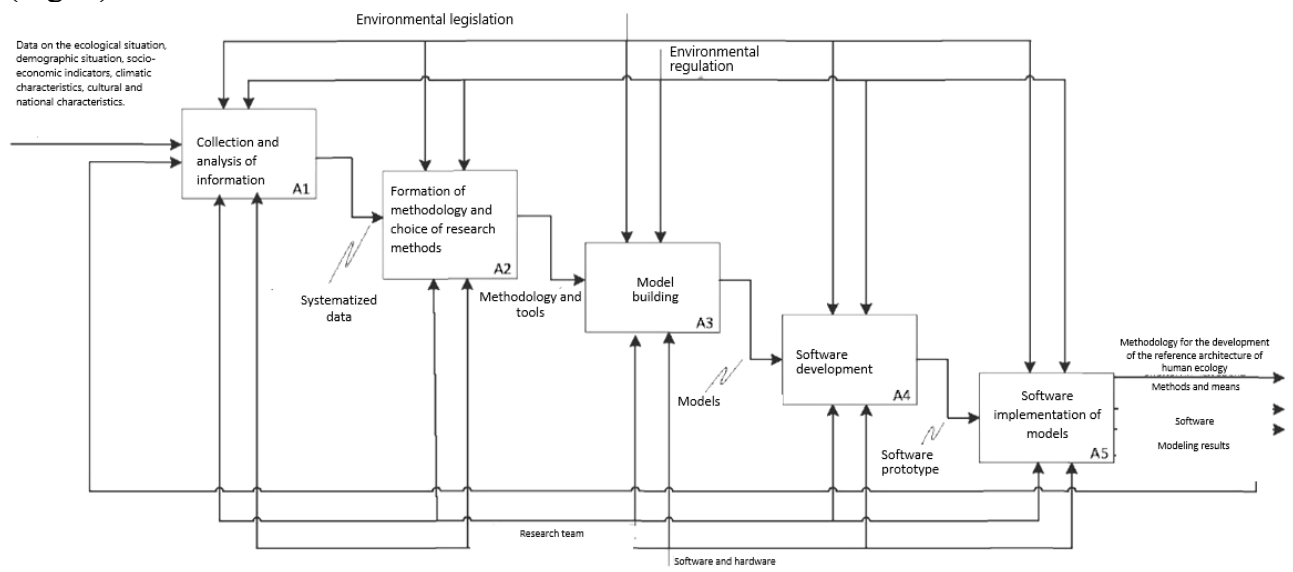

Fig. 2. Decomposition of the context diagram "Designing the reference architecture of human ecology".

Thus, the process of designing the reference architecture of human ecology includes a sequence of interrelated stages and each of them is a complex system of actions, the analysis and modeling of which are the tasks of subsequent research within the framework of the project to create the reference architecture of human ecology.

\section{Analysis of the main ERS data sources for the DEM development (Results and Discussion)}

Based on the results of the study carried out using the methodology of the function oriented modeling and a specialized software tool, the following statements can be made:

1. The developed models made it possible to systematize information about the design process of the reference architecture of human ecology, to identify its main stages and the 
results of their implementation, which is the necessary information for effective project management and software development.

2. The use of the function oriented approach to modeling business processes in the design and development of the reference architecture of human ecology seems promising, since it allows not only to detail and visualize activities in the form of a sequence of interrelated works, but also to serve as the basis for the development of appropriate software.

\section{Conclusions}

In the context of rapid urbanization of society, population growth and the development of world economic potential, problems related to the quality of life of people, which depend on many different factors and are the subject of study of human ecology, are becoming more and more urgent. The problems of human ecology are given close attention not only by scientists, but also by the world community as a whole. However, these problems are investigated separately and there are no uniform scientifically substantiated characteristics of the level of the state of human ecology, methodology, tools for their obtaining and processing means.

The paper presents one of the approaches to the design of the reference architecture of human ecology based on the application of the functional modeling methodology, which makes it possible to systematize the activity presenting it in the form of a sequence of works connected by information flows and transforming them. The set of functional models allows not only to systematize work on the design and development of the reference architecture of human ecology, but also to become the basis for creating an information system which in the future will automate the processes of creating the architecture of human ecology for individual regions, countries or cities and its comparison with the reference one.

\section{References}

1. V. Kh. He, I. N. Tkachenko, D. E. Skripnichenko, A. V. Dubinin, Bulletin peoples' friendship institute of the caucasus, Economic sciences, 2(42), 15 (2017)

2. Yu. S. Voronkova, E. V. Astashkina, Countering environmental crimes in the framework of the year of ecology in the Russian Federation in 2017, 219 (2017)

3. O. N. Luchko, S. Kh. Mukhametdinova, Modern science: problems and prospects for development: III International Scientific and Practical Conference: in 3 parts, Omsk, 6 (2019)

4. S. Mukhametdinova, E. Tyumentseva, O. Luchko, B. Irmulatov, Proceedings of the Ecological-Socio-Economic Systems: Models of Competition and Cooperation (ESES 2019), Series: Advances in Social Science, Education and Humanities Research, 392, 110 (2020)

5. A. G. Madzhuga, G. N. Kazantseva, L. M. Kashapova, N. V. Bagiyan, Scientific notes of the Russian State Social Universit, 19, 2(155), 62 (2020)

6. S. M. Kurbatova, L. Yu. Aisner, A. G. Volkova, IOP Conference Series: Earth and Environmental Science, 548(5), 052075 (2020)

7. A. Cai, J. Wang, I. MacLachlan, L. Zhu, Journal of Environmental Planning and Management, 63(13), 2357 (2020)

8. V. I. Drozhinov, V. P. Kupriyanovsky, D. E. Namiot, S. A. Sinyagov, A. A. Kharitonov, International Journal of Open Information Technologies, 5(3), 19 (2017) 
9. V. L. Makarov, A. R. Bakhtizin, G. L. Beklaryan, A. S. Akopov, National interests: priorities and security, 15, 2(371), 200 (2019)

10. L. Jung-Puo, H. Mei-Chih, Technological Forecasting and Social Change, 89, 80 (2014)

11. O. V. Maksimchuk, T. A. Pershina, Sociology of the city, 2, 33 (2014)

12. F. Shentaler, The Business processes, Modeling languages, methods, and tools, Moscow, 264 (2019) 